\title{
Service Ecosystem Emergence from Primitive Actors in Service Dominant Logic: An Exploratory Simulation Study
}

\author{
Satoru Fujita \\ Hosei University \\ fujita_s@hosei.ac.jp
}

\author{
Claudia Vaughan \\ University of Hawaii at Manoa \\ cmv815@hawaii.edu
}

\author{
Stephen L. Vargo \\ University of Hawaii at Manoa \\ svargo@hawaii.edu
}

\begin{abstract}
Service-dominant ( $S$-D) logic has been proposed as a theoretical foundation for understanding economic exchange and value cocreation from a service-forservice perspective. In the $S$-D logic framework, all economic entities are commonly represented as resource-integrating, service-providing actors, relying primarily on "operant" resources, such as skills and knowledge. Service exchange is coordinated by institutional arrangements, which form the bases of service ecosystems, the unit of analysis of value cocreation. Institutional arrangements and service ecosystems emerge from the resource integrating and service-exchanging activities of the actors. This paper reports a preliminary investigation of the emergence of these structures from basic actor relationships, through agent-based simulation. The simulations under different conditions show that a collection of agent interactions generates systemic behavior typical for service ecosystems. This paper also suggests directions for future research.
\end{abstract}

\section{Introduction}

The surge in interest and attention to service research and service innovation within the last decades has been profound [12]. Notably, initiatives such as the launch of service science by IBM [8] reflect the growing importance of the service concept to academics and practitioners alike, its significance to understand the business practices and activities of leading-edge firms and a reorientation of service as a process fundamental to economic exchange [12]. This development has further gained traction through the growing adoption of S-D logic [11], a framework that proposes "service" (usually singular) - a process of using ones resources for the benefit of and in conjunction with another party - as the fundamental basis of exchange [12]. Maglio and Spohrer suggested S-D logic as the philosophical foundation of service science, a scientific research domain that seeks to understand how entities within service systems exchange competences along various dimensions [8]. They argue that the "key to understanding the nature of these sharing arrangements lies in the distribution of competencies among entities and the value propositions that connect [them] [8]".

In S-D logic, service, as defined above, represents a transcending concept to goods and services, highlighting that economic exchange is primarily about the activities that actors do for others and want done for themselves. Goods are merely a service-distribution mechanism, and thus all economies are fundamentally service economies [7][11][12][13]. This view is relatively orthogonal to the more traditional approach rooted in neoclassical economics, in which exchange and value creation are understood in terms of goods tangible firm output embedded with value [12]. Consistent with others [11] we refer to such goodscentered thinking as goods-dominant (G-D) logic. In G-D logic, efficient production and distribution of goods are the primary concerns of business enterprises, whereas "services" (usually plural) are viewed either as intangible, somewhat inferior goods, or add-ons used to enhance the value of a good. [12].

S-D logic views all actors as generic [14] - that is, it disregards the producer-consumer divide, since all market participants mutually engage in serviceproviding and resource-integrating activities, central to value cocreation. More generally, S-D logic holds that the assignment of predefined roles to market actors unduly restricts our understanding of the mutually interdependent activities of actors from which roles and other institutional structures emerge. S-D logic seeks to understand markets and, at a higher level, whole economies, as a result of these mutual exchange activities and institutional structures that make up service-ecosystems -- relatively self-contained, selfadjusting systems of resource-integrating actors connected by shared institutional arrangements and mutual value creation through service exchange [16]. Arguably, this simplified view allows a clearer vision and understanding of the systemic nature of value 
creation, by examining the emergence of macro-level phenomena. In particular, researchers started to investigate the source of macro-level phenomena in terms of micro-level interactions [5]. That is, to examine how higher level structures emerge from the interplay and interaction of many individual actors. Emergence is often broadly defined as a property of a system that is not present in its parts, but that arises from their interaction (usually associated with serendipity, unexpected consequences, etc.). These emerging phenomena are also thought to form a "subset of the vast (and still expanding) universe of cooperative interactions that produce synergistic effects of various kinds" [2].

At the same time, multi-agent researchers in artificial intelligence have investigated the nature of emergent behaviors from agent interactions. A computational agent is an autonomous entity that occupies a space in an artificial world; acts and can interact with other agents and cooperate with them to perform a task. Especially in a large-scale agent simulation, a collection of agents generates unexpected patterns of behavior, as do ants in a colony. Researchers have argued that agent-based modeling (ABM) - a computational method that enables a researcher to create, analyze, and experiment with models composed of agents that interact within an environment [4] - is as of now the primary quantitative method for the direct observation and study of emergent phenomena [5].

In this paper, we conduct preliminary computational experiments, in which we represent the generic actors and their operant resources in an agentbased model, and examine their behavior in agentbased simulations. In the simulations, generic actors are represented by agents, who do not have explicit, predefined roles at the beginning, such as fishermen, farmers, and marketers, but instead get identified with such roles and specific locations in their environment over time as a result of dynamic environmental conditions and evolving skills (influenced by opportunities and experiences) brought about by engaging in service-for-service exchange. The agents gradually form a cooperative society that is consistent with important features of S-D logic's service ecosystem.

Section 2 briefly introduces some foundational concepts and terminologies of S-D logic, and Section 3 describes how these can be mapped to an agent-based model. Section 4 demonstrates some rudimentary simulation scenarios and presents their results. Section 5 discusses several issues surrounding an S-D logicbased service simulation, before section 6 concludes with some final remarks.

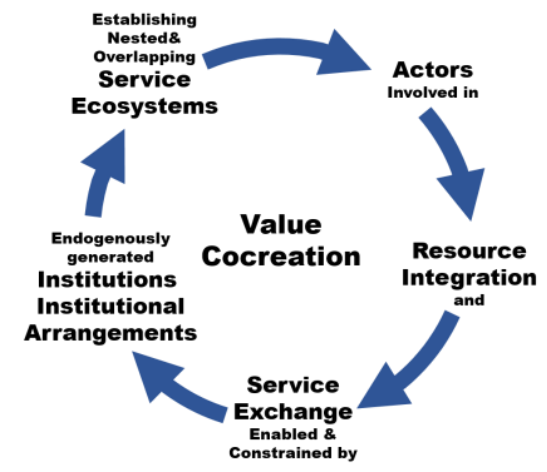

Figure 1. Core processes of service dominant

\section{Service Dominant Logic}

\subsection{Foundational Concepts}

Vargo and Lusch first introduced S-D logic in a paper in 2004 [11], in which they proposed an inverted interpretation of economic activities traditionally explained from a goods-dominant and firm-centric viewpoint [6][13][16]. The authors viewed these activities as service, a process in which multiple actors use their resources for the benefit of and in conjunction with another party for value cocreation. In S-D logic the distinction between producer and consumer becomes unnecessary, as does the assignment of any other predefined role (e.g. fisherman) to guide and explain an actor's activities. Instead, S-D logic emphasizes skills and abilities, which allow actors to be participants (service-providers and receivers) in a systemic value cocreation process. From the interplay of environmental conditions and repeated exchange experiences, institutions emerge, which enable actors to specialize in the use of their knowledge and skills and thus adapt their ability to and simultaneously shape their surrounding environment (an emerging service ecosystem).

Resources are thus not limited to materials but, in particular, include human skills and knowledge, to act. S-D logic divides types of resources into two categories: operand, such as natural, material resources and operant resources, such as knowledge and skills. Importantly, in S-D logic operant resources are primary, operand resources secondary [11].

Institutions, such as rules, customs and norms, are important for one actor to offer its service to another actor. The actors share various types of institutions, which on the one hand enable and on the other hand constrain their exchange. For example, they allow actors to act in the way that worked before and therefore prevent continuous trial and error when engaging in service exchange (without designing the details from scratch). 


\subsection{Value Cocreation Processes}

The basic concepts in S-D logic are interdependent; that is they are related to each other through cyclic processes of value cocreation, as shown in Fig.1 [17]. Actors are fundamentally not different from each other, but vary in their operant resources (e.g., skills and abilities). Service consists of the application of multiple resources (obtained from private, public and market sources) and are gathered and integrated by actors. Actors exchange service to satisfy their own requirements for living by helping others (thus increasing the viability of the system).

For service exchange to occur, actors depend on rules, called institutions. Some of these institutions are formalized (e.g. laws) and thus appear to be externally given, while others exist informally and silently emerge. All of them are, however selectively applied. These service activities, over time, stabilize value cocreating practices, resulting in discernible patterns. All emerge from actors' activities. In S-D logic, an interdependent structure is called a service ecosystem, conceptualized in terms of reciprocally serviceproviding actors, coordinated by institutions. Value cocreation processes are recursive and change institutions and ecosystems dynamically. Such ideas of dynamic institutions and ecosystems play an important role in innovation theory. 'The consideration of a service-ecosystems approach for innovation emphasizes that the maintenance, disruption, and change of institutions (i.e., institutionalization) are always co-creational processes in which actors try to resolve the nested contradictions and inconsistencies that are foundational to all institutional arrangements [15].' A broader perspective highlights the cocreated and systemic nature of value [18] and that institutional complexity drives the next innovation [10]. Such dynamism is embedded in value cocreation and service ecosystems -- relatively self-contained, self-adjusting systems of resource-integrating actors connected by shared institutional arrangements and mutual value creation through service exchange [15].

\section{Agent Based Modeling for S-D Logic}

The purpose of this research is to initiate the examination of emerging institutions and service ecosystems in value cocreation processes under the basic tenets of S-D logic. In value cocreation processes, many actors, resources and institutions dynamically interact with each other and allow for the performance of valuable activities in a society. Therefore, entire phenomena in a society are built up from many seemingly autonomous actors. Agent based modeling $(\mathrm{ABM})$ is appropriate to quantitatively express and analyze such group activities of autonomous actors from both micro- and macro-level viewpoints. Bonabeau discussed ABM as a method for simulating human systems and said that ABM works effectively when the interactions between the agents are complex, nonlinear, discontinuous or discrete, each agent is potentially different, and the population is heterogeneous [1]. From an S-D logic perspective, an actor can be directly mapped onto an ABS agent that has various types and levels of operand and operant resources and achieves individual goals by interacting with other agents. Therefore, ABM is likely a suitable methodological approach for exploring the development of economic activity within societies consistent with the S-D logic framework.

Negahban surveyed the use of agent-based simulation in marketing research and summarized that the major application of agent-based simulation is in the analysis of consumer behavior and advertising effects [9]. These works are helpful to design and observe actor communities in S-D logic. Fujita analyzed a price and market formation process under S-D logic using agent based simulation [3]. He formally defined the terms in S-D logic as parameters in agents. For example, he introduced 'capability' to express a level of operant resource. This paper follows these terminologies.

In order to examine the explanatory power of S-D logic, we keep the background setting very simple.

Actors reside on the land. In order to keep living, they need protein and carbohydrate.

We define some of the main concepts of S-D logic in the agent-based simulation as follows:

Actor

Actors are agents and therefore the main players in the simulation.

Operand Resource

Fish is a resource that provides protein.

Wheat is a resource that provides carbohydrates.

Land is a locational resource.

Operant Resource

Catching fish is a skill of an actor.

Growing wheat is a skill of an actor.

Plentifulness is the fertility level of land. 


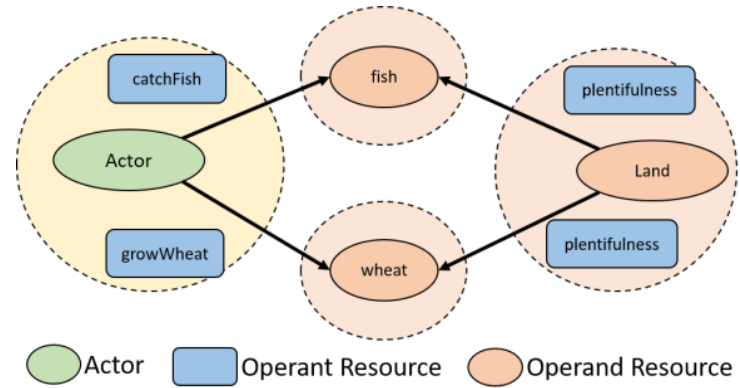

Figure 2. Actor and resources

Fig.2 represents the relationship among these terms. A yellow circle stands for an actor with two operant resources. Orange circles stand for operand resources, such as fish and wheat.

The simulation is controlled by periods. In every period, an actor must ingest a certain amount of protein and carbohydrate to keep alive; otherwise it dies. Each actor has a lifespan and at the end of the lifespan or when an actor dies in the middle of its life (due to a lack of protein or carbohydrate), a new actor is born. Properties from a long-lived ancestor are inherited based on inheritance rules, as follows:

Inheritance Rule

- If an actor lives until the end of its lifespan, its descendants probabilistically inherit its properties, such as capabilities (operant resources) and living location. Properties are slightly varied from the inherited ones by injecting Gaussian noise.

- If an actor dies in the middle of its lifespan because of a lack of protein or carbohydrate, its properties disappear from the world, and are not inherited.

Throughout the simulations no particular role, such as fisherman or farmer, is formally assigned to an actor. We use the terms fisherman and farmer only to denote their relative level of capability but not to impose in what activities they "should" engage. Initially, we only assign (randomly) capability values and locations to actors. More specifically, a capability consists of two parts, experience and effort. The experience value increases monotonically from birth to death, that is each time the actor successfully uses the corresponding operant resource. Stated somewhat differently, an actor that engages frequently in fishing increases its fishing capability. The maximum limit of the experience value is assigned as $\boldsymbol{N}\left(\mathbf{1 . 0}, \mathbf{0 . 2 ^ { 2 }}\right)$, where $\boldsymbol{N}\left(\boldsymbol{\mu}, \boldsymbol{\sigma}^{2}\right)$ stands for Gaussian distribution with average $\boldsymbol{\mu}$ and distribution $\boldsymbol{\sigma}^{2}$. Initially it is set as half of the maximum limit. The effort value is a ratio that splits an actor's workload (at each iteration or period) into how much time it invests in obtaining one or the other resource (thus the ratio of the use of his operant resources). The total amount of effort for using the operant resources that an actor holds is summed to 1 . Therefore, each effort value (for fishing and farming) is assigned with a uniform distribution between 0 and 1 at first, whereas the total amount of effort is canonicalized to 1.0. A particular capability (e.g. growing wheat) is calculated by multiplying an actor's experience value (e.g. to grow wheat) by the effort value to obtain the same resource. Coordinates $x$ and $y$ of the location are respectively assigned with a uniform distribution between 0 and the maximum width of the land, such as 1000 in the later experiments.

When the capability values and locations are inherited from an ancestor, the following is calculated in the later experiments:

$$
\begin{gathered}
\exp _{\max }=\mathrm{N}\left(\text { exp }_{\text {max }}^{\text {ancestor }}, 0.1^{2}\right) \\
\text { effort } \left.^{\text {ancestor }}, 0.1^{2}\right) \\
\text { loc }_{x \text { or } y}=\mathrm{effort}\left(\text { loc }_{x \text { or } y}^{\text {ancestor }}, \mathbf{1 0 0}^{2}\right)
\end{gathered}
$$

where the new experience value $\boldsymbol{e x p}_{\max }$ is inherited from $\boldsymbol{e x p}_{\max }^{\boldsymbol{a n c e s t o r}}$, the new effort value effortfrom effort $^{\text {ancestor }}$, and the new coordinates of the location $\boldsymbol{l o c}_{\boldsymbol{x} \text { or } \boldsymbol{y}}$ from $\boldsymbol{l o c}_{\boldsymbol{x} \text { or } \boldsymbol{y}}^{\text {ancestor. The sum of effort }}$ values for the operant resources is canonicalized to 1.0 afterwards.

In each period, actors expend effort to obtain protein and carbohydrate. If an actor finishes its lifespan or lacks for protein or carbohydrate, the actor dies and a new actor is born. The total population of actors is kept invariant. Over time the world gradually changes, partly due to actor deaths and births and partly because only good locations are inherited, whereas locations of actors with premature deaths are not. In this way an individual actor's location is not changed through the actor's lifespan, but the inherited actor is located close to the ancestor with the Gaussian distribution noise. Then, natural selection leads to a change in population distribution; it is an evolutionary mechanism.

\section{Emerging Ecosystem}

\subsection{Simple Scenarios}

This research explores the narrative of S-D logic shown in Fig.1. For this purpose, in the simulations, we prescribe very few specifications, which are consistent with the tenets of S-D logic, and then observe the structure that emerges. The first scenario is as follows:

\section{Scenario 1}

- The world is defined as a 1000x1000 grid field. For each grid, a set of plentifulness values for fish and wheat is assigned. 


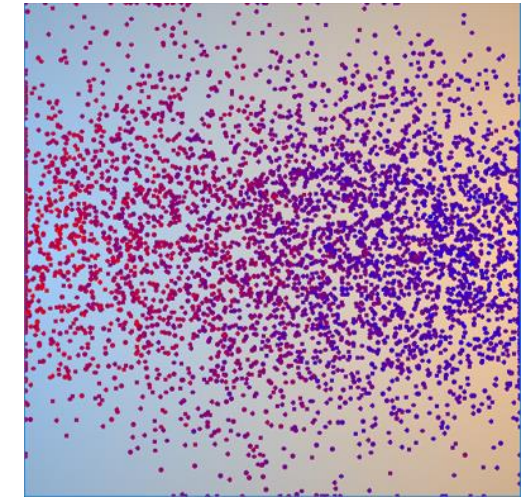

Figure 3. Final state in scenario 1

- An actor, located randomly at first, must ingest one unit of protein and carbohydrate in every period to keep alive.

- An actor's lifespan is limited to 80 periods.

- An actor has two types of capabilities, catching fish and growing wheat.

In this simulation, 5000 actors work in the grid world, and the plentifulness of the field for fish, namely $\boldsymbol{p}_{\boldsymbol{f}}$, and that for wheat, namely $\boldsymbol{p}_{\boldsymbol{w}}$ is given by the following equations.

$$
\begin{gathered}
p_{f}(x, y)=\frac{p_{0}}{\sqrt{x^{2}+(500-y)^{2}}} \\
p_{w}(x, y)=\frac{p_{0}}{\sqrt{(1000-x)^{2}+(500-y)^{2}}}
\end{gathered}
$$

The value of $\boldsymbol{p}_{0}$ (a controlled parameter of plentifulness) is tentatively set as 2200 . In this scenario, actors cannot exchange their service so they only obtain both fish and wheat by engaging in fishing and in farming. The exclusive reliance on one's own operant resources denotes self-service.

When the capabilities of actor $\boldsymbol{i}$ for fish and wheat are defined as $\boldsymbol{c}_{\boldsymbol{f}, \boldsymbol{i}}$ and $\boldsymbol{c}_{\boldsymbol{w}, \boldsymbol{i}}$, the amounts of fish and wheat obtained, namely $\boldsymbol{a}_{f, i}$ and $\boldsymbol{a}_{\boldsymbol{w}, \boldsymbol{i}}$ are calculated by the following equations:

$$
\begin{aligned}
a_{f, i}(x, y) & =c_{f, i} p_{f}(x, y) \\
a_{w, i}(x, y) & =c_{w, i} p_{w}(x, y)
\end{aligned}
$$

For example, if actor $\boldsymbol{i}$ located at $(500,500)$ has capabilities of $c_{f, i}=0.25$ and $\boldsymbol{c}_{\boldsymbol{w}, i}=0.25$, then actor $\boldsymbol{i}$ keeps living, because of $\boldsymbol{a}_{f, i}(500,500)=1.1$ and $a_{w, i}(500,500)=1.1$. However, this is a very severe condition, because if actor $\boldsymbol{i}$ is located at $(600,500)$, the amount of fish is now $\boldsymbol{a}_{f, i}(600,500)=0.92$, thus actor $\boldsymbol{i}$ dies because of a lack of protein.

Fig. 3 shows a population map of the actors after 1000 turns. The bottom-left is location $(0,0)$ and the top-right is location $(1000,1000)$. Actors are marked as small circles whose color is determined by the capability of an actor's operant resources. If an actor's capability value of growing wheat is very large (compared to catching fish), the color is red. If an actor has a larger capability value for catching fish, the color is blue. If the actor has equal capabilities its color is purple. The background color reflects the plentifulness of the location. The light blue color represents the land rich for fishing and the orange color represents the land rich for farming. Fig.3 demonstrates an interesting result: Fishermen, whose capabilities for fishing are higher (blue circles) live in the hillside; farmers, whose capabilities for farming are higher (red circles) live in the seaside. As noted, the terms "fisherman" and "farmer" are tentatively given and represent what actors primarily can do. No actor is a fisherman or farmer from birth.

Also as noted, initially, capabilities are randomly assigned to actors whereas subsequently actors inherit some capabilities from their ancestors according to the inheritance rule. Over time, such relative role distinctions among actors appear in the simulation. At first, this inverted distribution might cause surprise. However, it is reasonable that, without exchange, fishermen (actors with high fishing capability) can easily get the required amount of fish to live even if they inhabit the hill side and they can get the required amount of wheat (despite lower farming capability) because of the richness of the location for farming. Notably, actors do not specialize -- that is engage primarily in what they are good at but independently move to environments that support their most lacking capability.

The next scenario permits an actor to exchange its service with another actor, when the actor lacks fish or wheat.

\section{Scenario 2}

- In addition to scenario 1, an actor can now engage in service exchange with another actor, when the first actor lacks fish or wheat, and the second actor lacks the opposite resource. A pair-finding mechanism uses a simple random search, in which a resource-lacking actor randomly selects another actor on the grid who lacks the opposite resource. If the combined amounts of protein and carbohydrate for both actors exceed double the necessary amounts (2 units) the exchange succeeds. Otherwise, the actor continues to search for another actor. The periods to search are limited to five, but the distance between actor locations is not restricted.

In this scenario, two patterns are simultaneously observed as a result of the computational experiment. One resembles the one observed in scenario 1, in which fishermen live in the hillside and farmers live in the seaside. The other shows the opposite, fishermen 


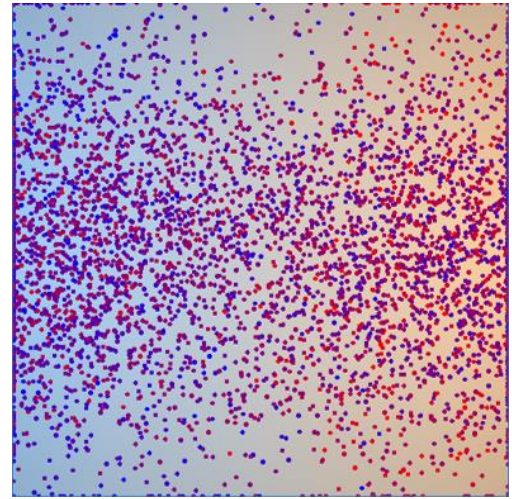

Figure 4. Final state in scenario 2

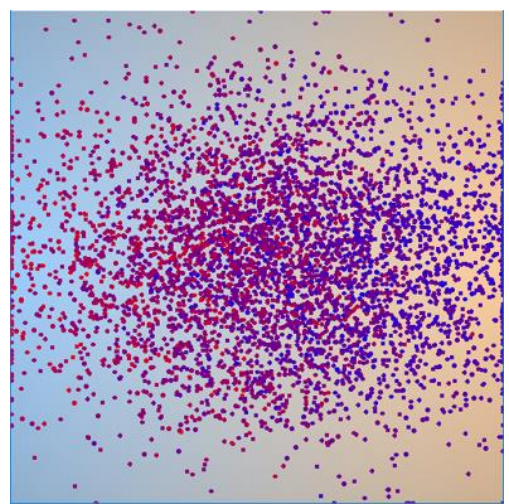

Figure 5. Exchange with limited distance

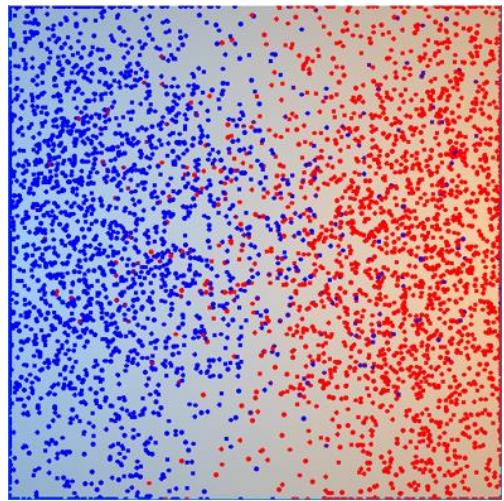

Figure 6 . Exchange with learning

now live in the seaside and farmers live in the hillside. Whereas the former actors are self-service actors who keep alive by relying only on themselves, the latter are actors specializing in their stronger capability while complementing their weaker capability by exchanging service with other actors, as shown in Fig.4.

We further investigate two modifications from scenario 2 . In the first one, we limit the permissible distance between actors in which they retain the ability to engage in service exchange. When the distance for exchange is limited to less than 500 unit lengths (measured in Euclid distance), over time the location of the population is shifted to the center of the field, as shown in Fig.5. On the one hand this is reasonable because a shorter spatial distance between actors increases the chance to exchange service with each other. On the other hand, this is consistent with S-D logic's claim that operant resources (e.g. fishing skills) are primary and operand resources (e.g. fish) are secondary. That is, capabilities and the ability to exchange them in the form of service are more important for survival (and thus location of the actors) than the plentifulness of the land (e.g. fish).

In the second modification, we introduce a learning function to actors. When an actor obtains more fish and less wheat than its periodical requirement, and it exchanges successfully its additional output of fish with a farmer who has a surplus of wheat but lacks fish, then the first actor "learns" -- that is it increases its effort value for fishing and thus its fishing capability after the exchange. As a result, at the next turn, the actor obtains more fish (from increased fishing capability) than in the last turn, so it gradually becomes a stronger fisherman. More specifically, an experience factor in capability is incremented by the corresponding effort multiplied by 0.01 , and an effort factor is incremented in the following equation.

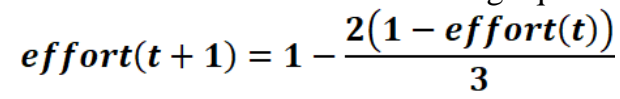

where $\boldsymbol{e f f o r t}(\boldsymbol{t})$ is the current effort value and effort $(t+1)$ is the next effort value. The total of both effort values (for fish and wheat) is canonicalized to 1.0 afterwards. Fig.6 shows the result. Actors with high fishing capabilities locate at the seaside and become even stronger fishermen, actors with high farming capability locate at the hillside and become even stronger farmers. By allowing exchange, specialization in a particular activity at a suitable location is so dominant that no purple (nonspecialized) actors exist.

\subsection{Exchange Scenario}

In the last section, we introduced service exchange, but it was limited to a sub-function (not a separate operant resource) of fishing and farming. This section introduces the exchanging skill as an independent operant resource that has a capability value and a learning function. 


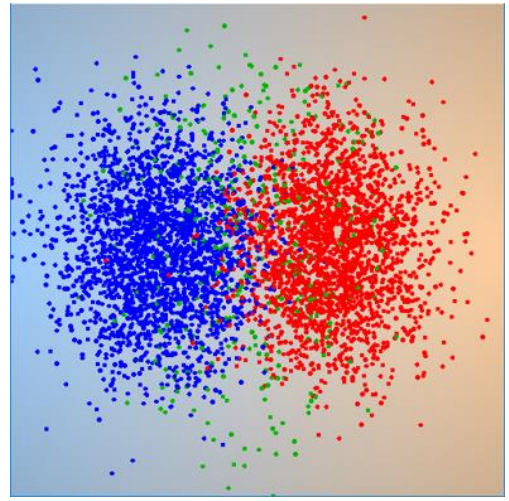

Figure 7. Final state in scenario 3

Scenario 3

- In addition to the two capabilities of scenario 1 , an actor possesses an exchanging capability.

- When an actor lacks both fish and wheat (thus it lacks enough of both operant resources), the actor applies its exchanging capability to find other actors who do not have fish enough to live and wheat enough to live. The actor obtains the amount of the insufficient operand resource in repeated exchanges for the amount of the superfluous resource multiplied by a specific exchange rate.

- As a learning function, the actor who initiates the service exchange increases its exchanging capability, the actor who obtains fish in exchange for wheat increases its growing-wheat capability, and the actor who obtains wheat in exchange increases its catching-fish capability.

The exchange rates are determined dynamically. For example, when two actors directly exchange their insufficient resource to complement each other, they exchange resources without observing an exchange rate. When an actor behaves as a marketer who exchanges resources with many actors, the actor sets its own exchange rate. If the marketer succeeded in the exchange, it makes the rate go up, otherwise it makes the rate go down.

In this scenario, three types of specialization are observed: fishing, farming, and exchanging. Fig.7 shows an example of the convergent state of the population distribution. The exchangers (green), are located at the middle of the field, and mediate the exchange of fish and wheat between specialized fishermen (blue) and farmers (red).

Additionally, interesting transient states are observed, one of which is shown in Fig.8. Here many fishermen first live in the hillside, and then move to the seaside. Fig.9 shows the history of the population. The $\mathrm{X}$-axis represents the passing of time, and the $\mathrm{Y}$-axis represents a population (measured in the distribution of capability levels in a certain skill, e.g., remember

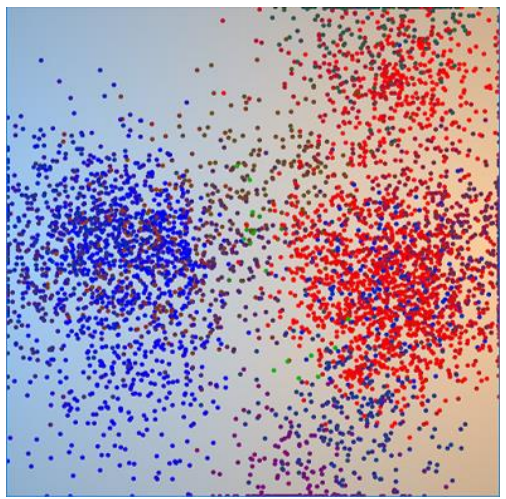

Figure 8. Transient state in scenario 3

farmers are simply actors high in farming skills). The

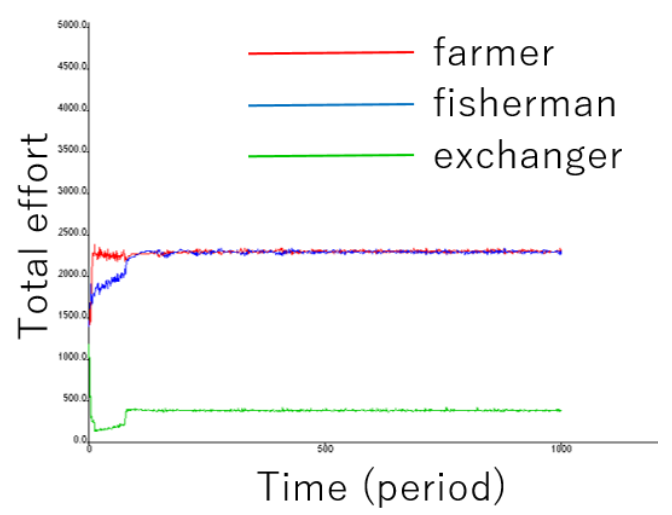

Figure 9. History graph in scenario 3.

colors indicate the types of actors. In this graph, a few exchangers exist in the early stage, and over time their population increases (that is exchanging capability proliferates). This demonstrates that, under conditions in which effort is limited, and knowledge about successful exchange is increasingly institutionalized (learning) actors shift to focus on one particular service activity and a new role (exchanger/marketer) emerges.

\subsection{Resource Integration Scenario}

In the previous sections, fishing and farming respectively required single operant resources, such as catching fish or growing wheat, without specifying resource integration. However, actual service often comes about through the integration of several resources. For example, fishing service is supported by the operant resources of finding and catching fish. If either of them is missing, an actor fails to obtain fish. We assume here that resource integration is realized by calculating the minimum amount of capability of the constituent resources; therefore the minimum resource becomes a bottleneck to the whole process. 


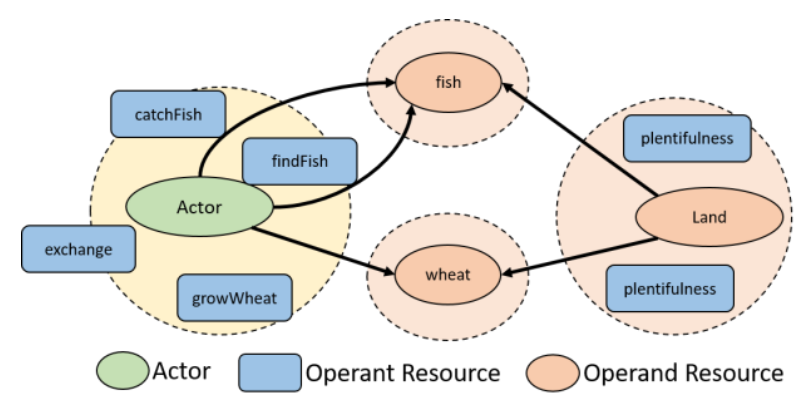

Figure 10. Resource integration

Scenario 4

- In addition to scenario 3, an actor possesses a finding fish capability. Resources of finding and catching fish are mutually dependent and integrated into obtaining fish. The minimum capability value of all constituent resources is calculated to determine the amount of fish obtained through the integrated process.

In this scenario, three types of fishermen are observed; fishermen specializing in finding fish, catching fish, and doing both (no specialization). Fig.10 shows the relationship among all resources and actors in scenario 4 .

We observe an interesting transient state. In the early stage, actors specialized in "finding fish", (yellow circles), and "catching fish" (blue circles) as shown in Fig.11. Then they increasingly become non-specialized fishermen (gray circles) as shown in Fig.12. Fig.13 shows the history of the population, in which a nonspecialized actor is represented as a magenta line. The number of non-specialized fishermen decreases in the early stage, then increases. This might be because the functions of finding and catching fish are linear, thus they are merged into a simpler solution that requires less exchange (i.e., transaction) cost. Stated somewhat differently, if the finding and catching fishermen uphold their different roles in the field, they need to pay additional cost to integrate their partial services. On the other hand, if a fisherman who possesses both resources lives as a non-specialized fisherman, he does not incur the additional cost to exchange. As a modified scenario, when an advantage of non-linear performance gains to separately specialized operant resources was given, such as finding and catching fish, the population of the separately-specialized actors dominated over the population of non-specialized actors.

\section{Discussion}

This research presents preliminary work to observe emergent behaviors under the conditions consistent

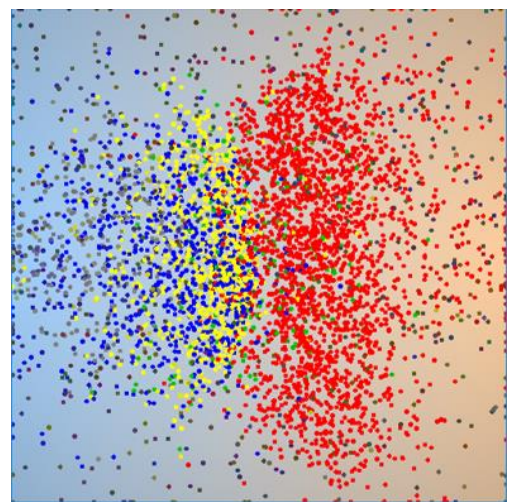

Figure 11. Transient state in scenario 4

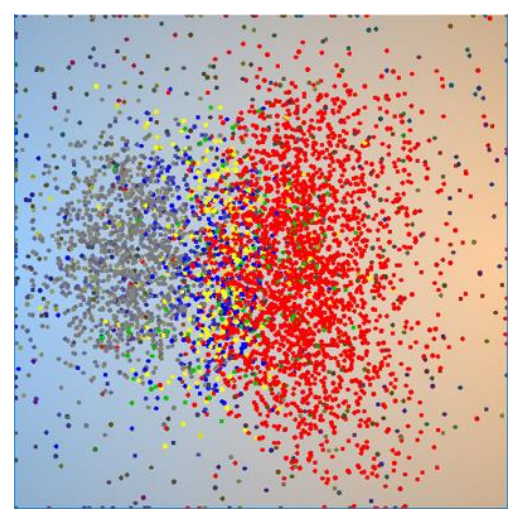

Figure 12. Final state in scenario 4

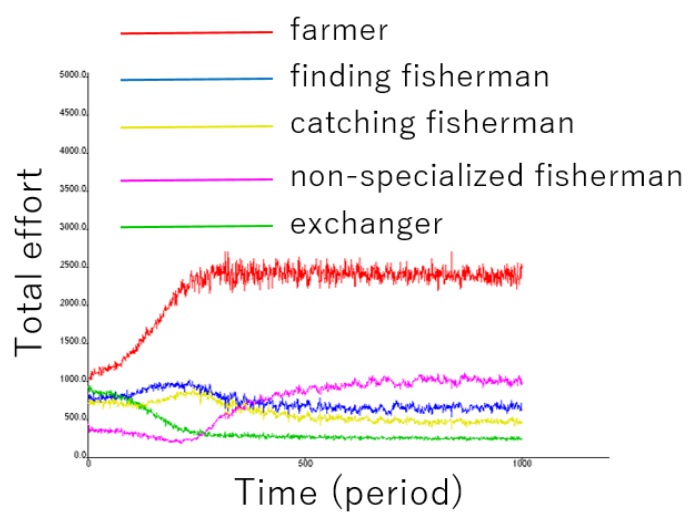

Figure 13. History graph in scenario 4

with S-D logic. The purpose of this paper was to observe emerging higher order structures (institutions) and, at an even higher-level, service ecosystems, from lower order elements (e.g. resources and service exchange).

Initially, actors had two types of operant resources, catching fish and growing wheat, but lived without service exchange. This simulation generated a pattern where each actor's location and activities were solely dependent on a match between an actor's operant resources and the operand resources of the 
environment (fish and wheat). There was no relationship between the locations and capabilities of actors to each other. In short, actor behavior remained autonomous and did not turn systemic.

In the case of allowing for resource (service) exchange, actors specialized into what they could do best (e.g. actors with high fishing capability moved to the rich fishing areas). Specialization is a first step in the process of institutionalization and leads to the emergence of roles and interdependence among actors. From a spatial point of view, this interdependence and thus systemic behavior was particularly pronounced when distance to exchange was limited. In this case actors bundled closely together, highlighting that the resourceness of land (operand resources) was secondary to the ability to exchange service, suggesting the emergence of a service ecosystem. When exchange was not limited by distance and actors could remember successful exchange (learning function), we observed over time an increasingly better match between environmental conditions (plentifulness of fish and wheat) and actor specialization. In other words exchange became institutionalized and selfservice actors largely disappeared.

The institutionalization of exchange was even more visible when we specified exchange as a separate capability, which over time led to the emergence of a new role "the exchanger" in the service ecosystem. Notable is that this role emerged and persisted despite actors' continuing ability to engage in direct servicefor-service exchange. Thus, through repeated periods of service exchange, three roles had institutionalized and actors behaved interdependently in time and space.

Adding service integration features, we could observe more complex behaviors in the transient states, such as transition and re-stabilization. In particular, in the long term, resource integration only led to more specialization (and thus systemic behavior) when the outcome of exchange was non-linear and thus created a combined advantage. This suggests an important value cocreation aspect of S-D logic.

As noted, service ecosystems are defined as relatively self-contained, self-adjusting systems of resource-integrating actors connected by shared institutional arrangements and mutual value creation through service exchange [15]. Our computational experiments realized such dynamic and self-contained features of service ecosystems over time. Very basic rules generated various institutions, and increasingly led to the emergence of an ecosystem, which then redefined and re-produced the local institutions of the exchange, and allowed it to change dynamically. This paper therefore supports the dynamic processes based on the principles of S-D logic, as shown in Fig. 1.
Our research also suggests that "cocreation", the cooperative activities among actors is a natural phenomenon and gives an agent society the power to obtain emerging properties (or in S-D logic terms "increases the viability of a system").

Negahban proposed ABM as suitable for the analysis of consumer behavior [9]. We extend this line of thinking by adopting the agent framework to both consumers and producers; that is, to a generic actor world. Just as in the real world, actors autonomously select their jobs, often as a result of what they are good at. And in line with institutional and practice theory, actors' activities and behavior are influenced by what worked before (here the learning function).

Furthermore, we adopted an evolutionary approach, which ensured that successful features (properties of long-lived actors) persist through inheritance, just as generations in real societies (through institutions) preserve their knowledge and skills for their off spring. While generations changed gradually, the social behaviors sometimes converged (stabilized) and sometimes oscillated.

The current simulator has no mechanism to create new resources, new types of values, or new abstract patterns of service exchange. We are planning to expand the current model to a richer model that has more functions, but simultaneously keeps the original base simple. Identifying and investigating general mechanisms that organically expand and lead to the evolution of the ecosystem's inner functions is an important and critical issue to proceed on this research.

\section{Conclusion}

This paper presented some preliminary work towards the investigation of emerging serviceecosystems in agent-based simulation. In the simulation, we defined generic actors equipped with several operant resources and observed how several types of service ecosystems emerged as a result of evolutionary changes in the actor community. At first actors only worked for themselves; we call this selfservice. When we introduced exchanging skill into the simulation, the actors showed collaborative behaviors towards each other. As a result, the locations and populations of actors started to change dynamically. The introduction of service integration also changed the structure of the community. In the next steps, we expect to explore (1) how the change in structure impacts individual actions thus (2) how higher order emergence develops from the more complex interactions.. 


\section{References}

[1] E. Bonabeau, "Agent-based modeling: Methods and techniques for simulating human systems," Proceedings of the National Academy of Sciences, vol. 99, no. 3, pp. 72807287, 2002.

[2] P. A. Corning, P. A. The re-emergence of emergence: A venerable concept in search of a theory. Complexity, 7, 18-30, 2002.

[3] S. Fujita and Y. Kase, "Service market simulation based on service dominant logic," IEEE International Conference on Agents, pp. 31-36, 2016.

[4] N. Gilbert. "Agent-based models". Sage Publications, p.2, 2008 .

[5] S. W. J. Kozlowski, G. T. Chao, J. A. Grand, M. T. Braun and G. Kuljanin, Advancing multilevel research design capturing the dynamics of emergence. Organizational Research Methods, 16(4), 581-615, 2013.

[6] R. F. Lusch and S. L. Vargo, "The Service-dominant Logic of Marketing: Dialog, Debate, and Directions". Sharpe, 2006.

[7] R. F. Lusch and S. L. Vargo, "Service-Dominant Logic: Premises, Perspectives, Possibilities," Cambridge University Press, 2014.

[8] P. P. Maglio and J. Spohrer, "Fundamentals of service science" . Journal of the Academy of Marketing Science, $36(1), 18-20,2008$

[9] A. Negahban and L. Yilmaz, "Agent-based simulation applications in marketing research: an integrated review," Journal of Simulation, vol. 8, no. 2, pp. 129-142, 2014.
[10] J. Siltaloppi, K. Koskela-Huotari and S. L Vargo, "Institutional complexity as a driver for innovation in service ecosystems," Service Science, vol. 8, no. 3, pp. 333-343, 2016.

[11] S. L. Vargo and R. F. Lusch, "Evolving to a new dominant logic for marketing," Journal of Marketing, vol. 68, no. 1 , pp. $2-17,2004$.

[12] S.L. Vargo, and R.F. Lusch, "From goods to service(s): Divergences and convergences of logics." Industrial Marketing Management, 37(3), 254-259, 2008.

[13] S. L. Vargo and R. F. Lusch, "Service-dominant logic: Continuing the evolution," Journal of the Academy of Marketing Science, no. 36, pp. 1-10, 2008.

[14] S. L. Vargo. and R. F. Lusch. "It's all B2B and Beyond . . . : Toward a Systems Perspective of the Market," Industrial Marketing Management, 40 (2) 181-187, 2011

[15] S. L. Vargo, H. Wieland and M. A. Akaka, "Innovation through institutionalization: A service ecosystems perspective," Industrial Marketing Management, vol. 44, pp. 63-72, 2015.

[16] S. L. Vargo and R. F. Lusch, "Institutions and axioms: an extension and update of service-dominant logic," Journal of the Academy of Marketing Science, vol. 44, no. 1, pp. 523,2016

[17] S. L. Vargo and R. F. Lusch, "Service-dominant logic in 2025," International Journal of Research in Marketing, vol. 34 , no. 1 , pp. 46-67, 2017.

[18] H. Wieland, K. Koskela-Huotari and S. L. Vargo, "Extending actor participation in value creation: an institutional view," Journal of Strategic Marketing, vol. 24, no. 3-4, pp. 210-226, 2016. 